\title{
Terrestrial planet formation in low-eccentricity warm-Jupiter systems
}

\author{
M. J. Fogg and R. P. Nelson
}

Astronomy Unit, Queen Mary, University of London, Mile End Road, London E1 4NS, UK e-mail: [M. J.Fogg; R.P.Nelson]@qmul .ac.uk

Received 7 November 2008 / Accepted 30 January 2009

\section{ABSTRACT}

\begin{abstract}
Context. Extrasolar giant planets are found to orbit their host stars with a broad range of semi-major axes $0.02 \leq a \leq 6$ AU. Current theories suggest that giant planets orbiting at distances between $\simeq 0.02-2$ AU probably formed at larger distances and migrated to their current locations via type II migration, disturbing any inner system of forming terrestrial planets along the way. Migration probably halts because of fortuitously-timed gas disk dispersal.

Aims. The aim of this paper is to examine the effect of giant planet migration on the formation of inner terrestrial planet systems. We consider situations in which the giant planet halts migration at semi-major axes in the range $0.13-1.7$ AU due to gas disk dispersal, and examine the effect of including or neglecting type I migration forces on the forming terrestrial system.

Methods. We employ an $N$-body code that is linked to a viscous gas disk algorithm capable of simulating gas loss via accretion onto the central star and photoevaporation, gap formation by the giant planet, type II migration of the giant, optional type I migration of protoplanets, and gas drag on planetesimals.

Results. Most of the inner system planetary building blocks survive the passage of the giant planet, either by being shepherded inward or scattered into exterior orbits. Systems of one or more hot-Earths are predicted to form and remain interior to the giant planet, especially if type II migration has been limited, or where type I migration has affected protoplanetary dynamics. Habitable planets in low-eccentricity warm-Jupiter systems appear possible if the giant planet makes a limited incursion into the outer regions of the habitable zone (HZ), or traverses its entire width and ceases migrating at a radial distance of less than half that of the HZ's inner edge.

Conclusions. Type II migration does not prevent terrestrial planet formation. A wide variety of planetary system architectures exists that can potentially host habitable planets.
\end{abstract}

Key words. planets and satellites: formation - methods: $N$-body simulations - astrobiology

\section{Introduction}

Giant planets are thought to form in the cool, outer, regions of a protoplanetary disk (e.g. Pollack et al. 1996; Papaloizou \& Nelson 2005; Boss 2000), in roughly the region where Jupiter and Saturn are found in our solar system. However, numerous giant exoplanets have been found orbiting solar-type stars well inside the approximate position of their nebular snowline with semi-major axes from $\sim 3$ AU down to just a few stellar radii (Butler et al. 2006). The most extreme examples of these are the so-called "hot-Jupiters", orbiting within 0.1 AU and accounting for about a quarter of the known giant exoplanet inventory. Planetary migration may provide the best explanation for the presence of the hot-Jupiter population, in particular type II migration, where the giant planet has grown massive enough to open a gap in its protoplanetary disk and migrates inward in step with the disk's viscous evolution (e.g. Lin \& Papaloizou 1986; Lin et al. 1996; Ward 1997; Nelson et al. 2000). Giant exoplanets at intermediate distances, where eccentricities can be high, might be explained by mutual scattering of giant planets (e.g. Lin \& Ida 1997; Ford et al. 2001; Papaloizou \& Terquem 2001; Marzari \& Weidenschilling 2002), a combination of migration and scattering (Adams \& Laughlin 2003; Moorhead \& Adams 2005), or migration along with eccentricity excitation from the disk (Papaloizou et al. 2001; Goldreich \& Sari 2003; Ogilvie \& Lubow 2003; Moorhead \& Adams 2008).
In the case of migrating planets, the mechanism that terminates the migration and strands exoplanets at their present orbital radii is unknown. Migration-halting mechanisms that might work when the planet ventures close to the central star include tidally-induced recession caused by the star's rotation or Roche lobe overflow and mass loss to the star (Trilling et al. 1998), or intrusion by the planet into a central cavity or surface density transition in the disk, decoupling it from the evolution of the gas (Lin et al. 1996; Kuchner \& Lecar 2002; Masset et al. 2006; Papaloizou 2007). Halting migration further out, beyond the $\lesssim 0.1$ AU hot-Jupiter region, may require that giant planets form late in the lifetime of the gas disk and hence only have time for a partial inward migration before stranding at an intermediate distance when the gas is lost (Trilling et al. 1998). Disks around T Tauri stars are observed to last for $\sim 1-10 \mathrm{Myr}$ (Haisch et al. 2001) but disperse over a much shorter $\sim 10^{5}$ year timescale (Simon \& Prato 1995; Wolk \& Walter 1996): a behaviour that may result primarily by accretion of gas onto the central star combined with photoevaporative gas loss driven by the stellar UV output (Clarke et al. 2001; Alexander et al. 2006). Models of this stranding mechanism (Armitage et al. 2002; Armitage 2007), which can roughly reproduce the exoplanet semi-major axis statistics, have raised the possibility that fortuitous disk dispersal might also explain the presence of the hot-Jupiter population and imply that earlier formed giant planets could have been consumed by the cental star. 
If type II migration correctly accounts for the presence of the hot-Jupiter population then these giant planets must have traversed their inner systems at a time when gas was still present and before the completion of terrestrial planet formation. This prompted initial speculations that such systems would be likely to lack any terrestrial planets within their inner few AU (Armitage 2003), and since hot-Jupiters are not uncommon, they have been used to infer significant constraints on the abundance of habitable planets (Ward \& Brownlee 2000), and even their galactic location (Lineweaver 2001; Lineweaver et al. 2004). This view is contradicted however by recent models that have simulated the process of a giant planet migrating through an interior protoplanetary disk (Fogg \& Nelson 2005, 2006; Raymond et al. 2006; Fogg \& Nelson 2007a; Mandell et al. 2007; Fogg $\&$ Nelson 2007b). These find that solid material is not predominantly accreted by the giant planet or the central star; instead, solid bodies captured at interior mean motion resonances with the giant are shepherded inward an arbitrary distance before being randomly scattered into an external orbit. The net result after the migration is a partitioning of most of the original disk material into two remnants: a compacted remnant interior to the final orbit of the giant, which typically accretes in a short timescale to form hot-Earth or hot-Neptune planets; and an external disk of scattered bodies. The relative predominance of these outcomes has been shown to be sensitive to the strength of dissipative forces operating at the time of migration (Fogg \& Nelson 2005, 2007a,b) with scattering becoming increasingly prevalent in late migration scenarios when less gas is present. All these studies concur that a scattered disk of sufficient mass to support renewed planet formation is likely to be generated under a variety of conditions and that terrestrial planets should be commonplace in hot-Jupiter systems, rather than rare or absent.

One simplification common to these previous models is that the physical mechanism that actually halts giant planet migration is not specified or modeled. Type II migration is artificially halted when the giant planet has reached a preset final orbit and hence is not determined by the structure or evolution of the gas disk. Since these models stop migration close to the central star, whilst significant gas is still present, they appear most realistic in the context of a central gaseous cavity halting mechanism. The examples that come closest to implicitly assuming fortuitous gas disk dispersal as the halting mechanism are the late scenarios of Fogg \& Nelson (2007a,b) where gas densities have fallen to low levels and migration is decelerating (see Fig. 3 in Fogg \& Nelson 2007a, and Fig. 3 of this paper). However, the final hot-Jupiter orbits in these papers are still artificially imposed at $0.1 \mathrm{AU}$ and are not controlled self-consistently by the evolution of the gas.

Our previous model adopted a 1-D, viscously evolving, gas disk algorithm which simulates accretion onto the central star, annular gap formation in the vicinity of a giant planet, and selfconsistent type II migration; for the nebular parameters chosen, the mass of our gas disk exponentially declined with an e-folding time of 582000 years (Fogg \& Nelson 2007a). This sort of model runs into trouble when simulating the late stages of gas disk dispersal as it does not reproduce a final and abrupt $\sim 10^{5}$ year decline that would accord with observations. We have corrected this deficiency here by including a photoevaporation algorithm in our code that gradually erodes and removes mass from our gas disk. As shown by Clarke et al. (2001) and Alexander et al. (2006), this process has little effect on the evolution and structure of the gas disk at early times, but comes to dominate at later times once the rate of gas loss onto the central star due to viscous evolution falls below the photoevaporation rate. A rapid dispersal of the remaining gas follows, along with the cessation of any ongoing giant planet migration.

In this paper, we report on the results of a set of scenarios where giant planet stranding distances are no longer prescribed but which happen when migration runs out of steam at the time of the disappearance of the nebular gas. We therefore specifically assume and self-consistently model fortuitous gas disk dispersal as the mechanism that finalizes giant planets in their postmigration orbits. Terrestrial planetary formation in this context is of interest because, for a hot-Jupiter to strand at $\sim 0.1 \mathrm{AU}$, it must form and migrate late in the lifetime of the gas disk, when gas densities are lower and accretion in the inner system is at a more advanced stage than previously considered. In addition, a succession of later scenarios than this results in a succession of shorter migrations and larger stranding distances. This has allowed us to extend the scope of our study to model terrestrial planet growth in those "warm-Jupiter" systems that may have originated as the result of a late, partial, inward migration. In this paper we define a "warm-Jupiter" to be one orbiting with semi-major axis in the range $0.1<a<2.7 \mathrm{AU}$, where the outer limit coincides with the snowline.

The plan of the paper is as follows. In Sect. 2 we outline the additions to our model and the initial conditions of the simulations; in Sect. 3 the results are presented and discussed; in Sect. 4 we consider some caveats, and in Sect. 5 we offer our conclusions.

\section{Description of the model}

We model our systems using an enhanced version of the Mercury 6 hybrid-symplectic integrator (Chambers 1999), run as an $N+N^{\prime}$ body simulation, where there are $N$ protoplanets embedded in a swarm of $N^{\prime}$ "super-planetesimals" - tracer particles with masses a tenth of the initial masses of protoplanets that act as an idealized ensemble of a much larger number of real planetesimals and are capable of exerting dynamical friction on larger bodies (e.g. Thommes et al. 2003). The central star, giant planet, and protoplanets interact gravitationally and can accrete and merge inelastically with all other bodies. Superplanetesimals however are non-self-interacting but subject to a drag force from their motion relative to the nebular gas that is equivalent to the gas drag that would be experienced by a single $10 \mathrm{~km}$ radius planetesimal. Details of these aspects of our model are given in Fogg \& Nelson (2005).

We calculate the evolution of the nebular gas using a 1-D viscous disk model that solves numerically a modified viscous gas disk diffusion equation that includes the tidal torques exerted by an embedded giant planet (Lin \& Papaloizou 1986; Takeuchi et al. 1996) and have described its implementation in Fogg \& Nelson (2007a). The gas responds by depleting over time via viscous accretion onto the central star; opening up an annular gap centred on the giant planet's orbit; and forming a partial inner cavity due to dissipation of propagating spiral waves excited by the giant planet. The back reaction of these effects on the giant planet is resolved as torques which self-consistently drive type II migration. We model the possible effects of type I migration (Ward 1997; Papaloizou \& Larwood 2000; Tanaka et al. 2002; Tanaka \& Ward 2004; Cresswell \& Nelson 2006), where a tidal interaction with the gas disk is thought to exert an inward radial drift and strong eccentricity and inclination damping on protoplanets of $\sim 0.1-100 M_{\oplus}$, using a simple algorithm described in Fogg \& Nelson (2007b).

As well as modeling the dynamics of gaseous volatiles in our model, we also track the movement of presumed solid volatiles, 
such as water ice and hydrated minerals, by labeling all particles with a composition based on their original location in the disk and summing the composition of protoplanets as they grow. We assume a crude three-phase initial radial composition with rocky material originating at $<2 \mathrm{AU}$, material similar to chondritic meteorites between 2-2.7 AU, and trans-snowline material at $>2.7 \mathrm{AU}$. We do not assign an actual water mass fraction to these phases.

\subsection{Photoevaporation-driven disk dispersal}

A gas disk that viscously drains onto its central star undergoes a power law decline with a long drawn out dispersal in conflict with observations that final dispersal occurs over a timescale that is short compared with the disk age. Clarke et al. (2001) showed that this could be explained by including a model of photoevaporation of the disk driven by the diffuse UV flux from the central star (Hollenbach et al. 1994). Their results show that once the accretion rate onto the star declines to roughly equal the outer disk photoevaporation rate, the inner disk ceases to be resupplied from larger radii and rapidly drains onto the star. The formation of this inner cavity then permits direct UV illumination of the outer disk which disperses in turn in $\sim 10^{5}$ years (Alexander et al. 2006).

For our purposes we need only adopt a simple parameterization of this type of photoevaporation model and subtract from the right hand side of our disk diffusion equation (Eq. (7) in Fogg \& Nelson (2007a)) an extra term representing a disk wind:

$\dot{\Sigma}_{\mathrm{W}}=K\left(\frac{r_{\mathrm{g}}}{r}\right)^{2.5}, \quad r_{\mathrm{g}} \leq r \leq r_{\text {out }}$,

where $\dot{\Sigma}_{\mathrm{W}}$ is the rate of change of gas surface density due to photoevaporation, $r$ is radial distance, $r_{\text {out }}$ is the disk radius, and $r_{\mathrm{g}}$ is the gravitational radius: the distance beyond which ionized gas can become unbound from the star. The constant of proportionality $K$ depends directly on the disk's total photoevaporative mass loss rate $\dot{m}_{\mathrm{w}}$ :

$K=\dot{m}_{\mathrm{W}}\left(-2 \pi r_{\mathrm{g}}^{2.5} \int_{r_{\mathrm{g}}}^{r_{\text {out }}} r^{-1.5} \mathrm{~d} r\right)^{-1}$.

To fit with our pre-existing viscous gas disk model, we take $r_{\text {out }}=33 \mathrm{AU}$, and adopt $r_{\mathrm{g}}=5 \mathrm{AU}$ and $\dot{m}_{\mathrm{w}}=10^{-9} M_{\odot} \mathrm{yr}^{-1}$ which gives $K=1.4685 \times 10^{-12} \mathrm{~g} \mathrm{~cm}^{-2} \mathrm{~s}^{-1}$.

In Fogg \& Nelson (2007a,b) we assumed an initial condition of a minimum mass solar nebula model (Hayashi 1981), scaled up in mass by a factor of three $(3 \times$ MMSN), extending between 0.025-33 AU from a solar mass protostar, with an initial surface density profile of $\Sigma_{\mathrm{g}} \propto r^{-1.5}$ and a total mass of $0.039 M_{\odot}$. Having chosen an alpha viscosity of $\alpha=2 \times 10^{-3}$, we found that, after a short lived $\sim 10^{5}$ year period where $\Sigma_{\mathrm{g}}$ close to the star relaxes to a shallower profile, the mass of the gas disk declines predictably with an e-folding time of 582000 years. This behaviour is illustrated as the upper blue curve in the top panel of Fig. 1, which plots the nebular mass vs. time, and is compared with the red curve which illustrates the effect of including photoevaporation. It is evident that the two models only diverge slowly for the first $\sim 2 \mathrm{Myr}$, but thereafter the mass of the photoevaporating disk drops steeply and vanishes in just a few $\times 10^{5}$ years. The lower panel of Fig. 1, which plots the accretion rate onto the star $\dot{m}_{*}(t)$, shows that this transition in behaviour occurs around the time when $\dot{m}_{*} \approx \dot{m}_{\mathrm{w}}$.

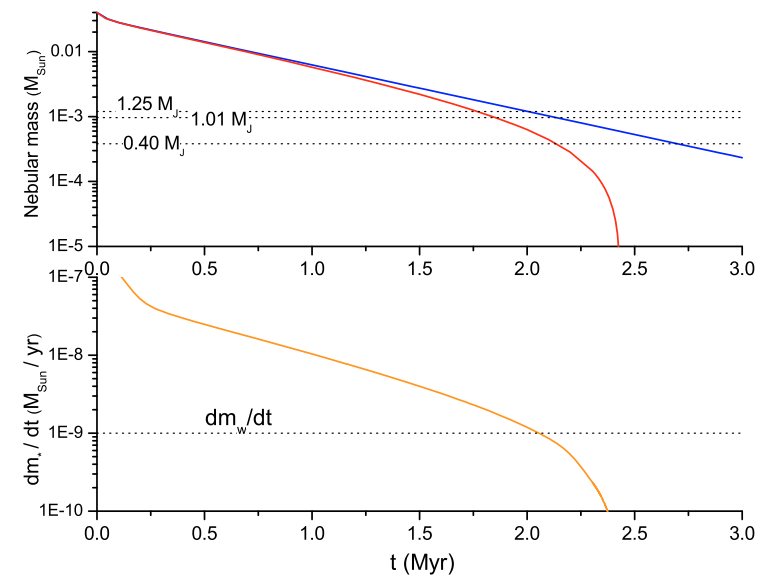

Fig. 1. Upper panel: mass of the nebular gas vs. time: the blue curve represents our former model where mass loss occurs solely via viscous accretion onto the central star; the red curve represents our new model including photoevaporation. Lower panel: accretion rate onto the central star $\mathrm{d} m_{*} / \mathrm{d} t$. The dotted horizonal line represents the photoevaporation rate $\mathrm{d} m_{\mathrm{w}} / \mathrm{d} t=10^{-9} M_{\odot} \mathrm{yr}^{-1}$.

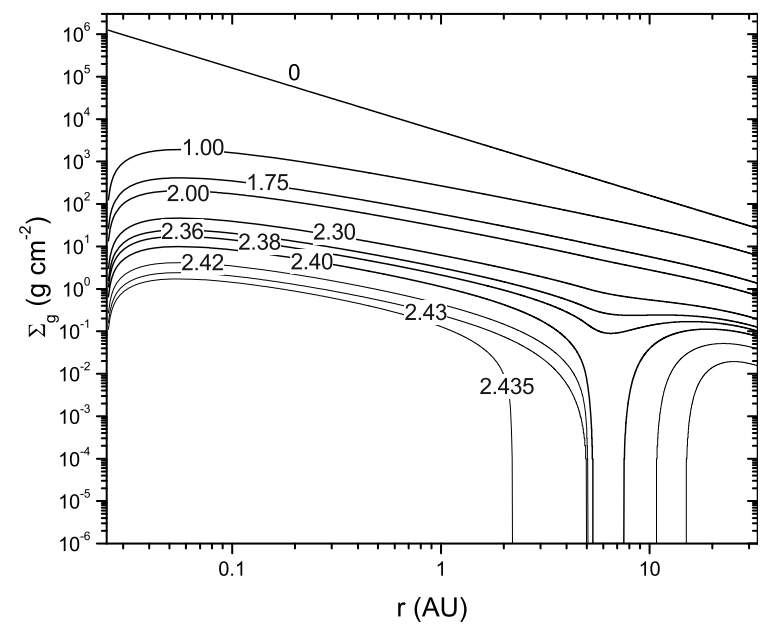

Fig. 2. Model gas disk surface density evolution. The uppermost curve is the initial condition; successive curves are labeled with their age in Myr.

The evolution of the gas disk surface density $\Sigma_{\mathrm{g}}(r, t)$ is shown in Fig. 2, where the uppermost curve represents the initial $\Sigma_{\mathrm{g}} \propto r^{-1.5}$ profile and the lower curve represent successively more evolved configurations. It can be seen that the evolution of the nebula speeds up after $\sim 2 \mathrm{Myr}$, with a gap at $r \approx r_{\mathrm{g}}$ starting to open up at $\sim 2.38 \mathrm{Myr}$, followed by an accelerated decline of the inner disk thereafter. This behaviour is qualitatively similar to that described by Clarke et al. (2001), with the exception that at late times our outer disk, which is truncated to a much smaller radius, is lost even more rapidly. This makes no difference to our mechanism for stranding giant planets as the divergence occurs when the nebular mass has already fallen below the level where it can drive migration. If giant planets strand well inward of $r_{\mathrm{g}}$ we found that they can act against the efficient draining of the last dregs of the inner disk onto the central star, slightly altering the picture given in Fig. 2. Again however, this effect is minor as it occurs at times when the gas is very thin and does not significantly delay the date of overall disk dispersal. 
Table 1. Data describing initial solids disk set-up.

\begin{tabular}{c|cc|c}
\hline \hline & Rocky zone & Icy zone & Total \\
& $0.4-2.7 \mathrm{AU}$ & $2.7-5.0 \mathrm{AU}$ & $0.4-5.0 \mathrm{AU}$ \\
\hline$M_{\text {solid }}$ & $9.99 M_{\oplus}$ & $24.65 M_{\oplus}$ & $34.64 M_{\oplus}$ \\
\hline$m_{\text {proto }}$ & $0.025 M_{\oplus}$ & $0.1 M_{\oplus}$ & \\
$N$ & 66 & 15 & 81 \\
\hline$m_{\text {s-pl }}$ & $0.0025 M_{\oplus}$ & $0.01 M_{\oplus}$ & \\
$N^{\prime}$ & 3336 & 2315 & 5651 \\
\hline$f_{\text {proto }}$ & 0.17 & 0.06 & 0.09 \\
\hline
\end{tabular}

\subsection{Initial conditions and running of the simulations}

We do not consider the earliest stages of planetesimal formation and runaway growth in our modeled systems and set the $t=0$ start date for our simulations to be $0.5 \mathrm{Myr}$ after the start of star formation. By this time, we assume that there is no more infall of gas onto the protoplanetary disk and the solids component of the inner disk has reached its oligarchic growth stage, where a succession of protoplanets, each being a few percent of an Earth-mass, have emerged from the planetesimal swarm in nearcircular orbits and with roughly equidistant spacing in units of mutual Hill radii (Kokubo \& Ida 2000). We assume a central star of $1.0 M_{\odot}$ with initial surface density profiles for gas and solids $\left(\Sigma \propto r^{-1.5}\right)$ taken from a minimum mass solar nebular model (Hayashi 1981), which is scaled up in mass by a factor of three $(3 \times$ MMSN) to provide enough mass beyond the nebular snowline for a giant planet to form before the loss of the nebular gas (Lissauer 1987; Thommes et al. 2003). We model the gas component of the protoplanetary disk between $0.025-33 \mathrm{AU}$, giving an initial mass of $0.039 M_{\odot}$, and compute its evolution with our photoevaporating viscous gas disk algorithm, as described in the previous section and in Fogg \& Nelson (2007a). We take the alpha viscosity of the gas to be $\alpha=2 \times 10^{-3}$, which gives a viscous evolution time at $5 \mathrm{AU} \simeq 120000$ years.

We model the solids component of the disk initially between 0.4-5.0 AU and assume a snowline at 2.7 AU beyond which the mass of solids is boosted by a factor of 4.2 by the condensation of ices. We generate the initial $N$-body components of the solids disk in an identical manner to that detailed in Fogg \& Nelson (2005) by starting with initial protoplanetary masses of 0.025 and $0.1 M_{\oplus}$ interior and exterior to the snowline respectively, spaced approximately 8 mutual Hill radii apart, with the remainder of the material inventory consisting of super-planetesimals with a fixed mass of $10 \%$ of the initial masses of the local protoplanets. Relevant data for the initial solids components are shown in Table 1 which gives, for zones interior and exterior to the snowline, values for the total mass of solid material $M_{\text {solid }}$, the number and mass of protoplanets $N$ and $m_{\text {proto }}$, and the number and mass of super-planetesimals $N^{\prime}$ and $m_{\mathrm{s}-\mathrm{pl}}$. The parameter $f_{\text {proto }}$, at the foot of Table 1 , is the mass fraction of the solids disk contained in protoplanets and we use this here as a rough measure of the evolution of the disk, taking $f_{\text {proto }}=0.5$ to denote the transition between oligarchic and chaotic, or "giant impact", growth regimes (Goldreich et al. 2004).

Our previous approach was to run our combined $N$-body and gas disk model, in the absence of a giant planet, from $t=0$ to a set of durations distributed between $0.1-1.5 \mathrm{Myr}$, in order to mature the disk to different ages and to generate a set of migration scenarios. At the end of each of these maturation runs, we then introduced a $0.5 M_{J}$ giant planet at $5 \mathrm{AU}$, after removing $0.4 M_{J}$ of gas from a local disk annulus to provide for the giant planet's envelope. This gas is assumed to have accreted on top of a $0.1 M_{J}$ solid core, composed of material deriving from beyond the outer boundary of our modelled solids disk. Re-starting the run at this point resulted in an inward type II migration of the giant planet which was allowed to continue until it reached our prescribed stranding radius of $0.1 \mathrm{AU}$. This approach is not appropriate here as we are specifically assuming gas disk dispersal as the stranding mechanism and therefore need to mature the disk to between the boundaries of a temporal window within which our giant planet can both accrete sufficient gas for its envelope and cease migration at $\gtrsim 0.1 \mathrm{AU}$. We located the lower limit of this window, the age where a $0.5 M_{J}$ giant planet introduced at $5 \mathrm{AU}$ will naturally cease migrating and come to rest at $\sim 0.1 \mathrm{AU}$, via experiments with the model and found it to be $t \approx 1.77$ Myr. By this time, the mass of the nebula has fallen to $M_{\mathrm{gas}} \approx 1.25 M_{J}$ which is shown as the upper dotted line in Fig. 1. The maximum possible upper age limit of the stranding window would be when $M_{\text {gas }}=0.4 M_{J}$, at $t \approx 2.13 \mathrm{Myr}$ (see the lower dotted line in Fig. 1), giving a window duration of $\sim 17 \%$ of the simulated disk lifetime. However, this limit would require the unrealistic condition of all the remaining nebular gas being accreted by the giant planet. Since we do not simulate the process of gas accretion onto the giant planet's core, we have arbitrarily restricted the upper age limit of the stranding window to $t=1.85 \mathrm{Myr}$ (see the middle dotted line in Fig. 1) by which time the mass of the nebula has fallen to $M_{\text {gas }}=1.01 M_{J}$, reducing the window duration to $\sim 4 \%$ of the simulated disk lifetime. We note that the model of Armitage et al. (2002) predicts a stranding window of duration $\approx 20 \%$ of the disk lifetime. This longer duration compared to ours is because they adopted a more slowly evolving disk model and were simulating the stranding of more massive giant planets which migrate more slowly. Clearly, variation of the many free parameters in a model such as this can produce a variety of stranding behaviours in simulations, but we have not considered these here as our main focus is on the effect that the giants' stranding distances have on the partitioning of the inner system disk and subsequent terrestrial planet formation.

The behaviour of $0.5 M_{J}$ giant planets launched into our model disks, between the lower and upper age limits discussed above, is illustrated by the solid curves in Fig. 3 and shows that stranding takes place between 0.13-1.71 AU. Also illustrated by the dashed curves in Fig. 3 are the migration trajectories of the giant planets in Scenarios III, IV, and V of Fogg \& Nelson (2007a) where migration takes place in a younger disk and was artificially halted at $0.1 \mathrm{AU}$ by a presumed inner disk cavity. The fastest of these (launched at $0.5 \mathrm{Myr}$ ) takes place at a time when the gas disk is still quite massive and completes its migration in the viscous evolution time of $\sim 120000$ years. Later scenarios entail longer migration times as the gas mass progressively declines and is less effective at driving migration. In order for giant planets to strand naturally, our present models require still later launch times, in a context where photoevaporation is starting to have a significant influence on the disk. Figure 3 shows that migration speeds are considerably slower and decelerate steadily until migration ceases after $t_{\mathrm{s}}=600000$ years for the farthest travelling planet and 470000 years for the planet that migrates the least. Migration in all these cases halts at $t \approx 2.4 \mathrm{Myr}^{1}$.

Thus, we generated the scenarios for this paper by running the model from its initial condition, without a giant planet

\footnotetext{
1 Note that the gap between the $t=1.82$ and 1.83 Myr curves is because of the substantial depletion of disk gas at these late times, dictating the removal of the quantity required for the giant planet's envelope from a wider annulus of the disk than previously.
} 


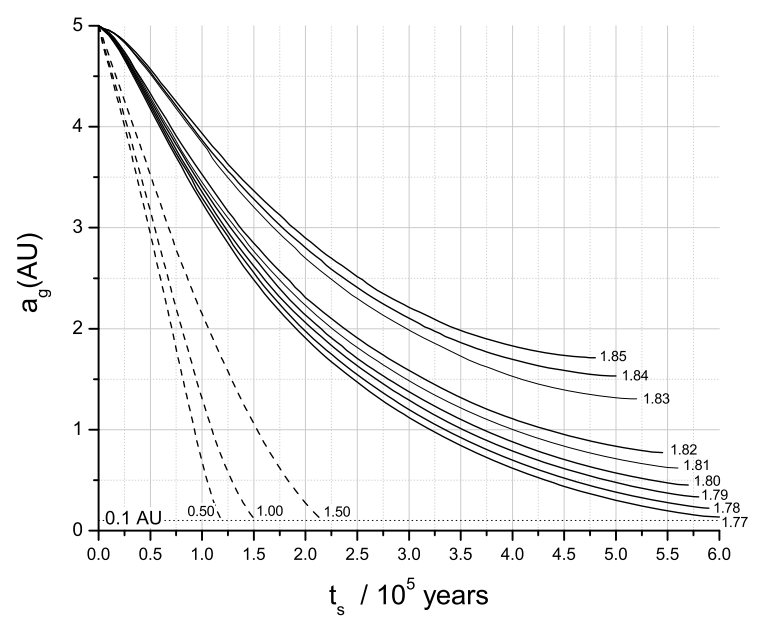

Fig. 3. Migration and stranding of $0.5 M_{J}$ giant planets in our present and previous models. Giant planet semi major axis in AU is plotted against $t_{\mathrm{s}}$, time normalized to the start of migration, in units of $10^{5} \mathrm{yr}$. Black curves show the behaviour of giant planets stranding naturally due to gas disk photoevaporation. Numeric labels are launch dates: the respective disk ages $t / \mathrm{Myr}$ at which the giant planet is introduced. Dashed curves show giant planet migrations in Scenarios III, IV, and V from Fogg \& Nelson (2007a), where migration was artificially halted at $0.1 \mathrm{AU}$.

present, to mature the protoplanetary disk to a minimum of $t=1.77 \mathrm{Myr}$ and then in successive 10000 year increments to $t=1.85$ Myr. Given that the reality of strong type I migration is controversial, and to bracket the range of possibilities, two parallel sets of scenarios are generated: one with no type I migration forces (Run Set $\mathbf{A}$ ) and the other with type I migration and eccentricity and inclination damping set at the maximum rate determined by Eqs. (1) and (2) in Fogg \& Nelson (2007b) (Run Set $\mathbf{B})$. To accommodate this total of 18 simulations, a change from our previous scenario ID system is also required. Thus, Roman numerals are substituted with Arabic numerals and labelling is generated by the following formula: Scenario ID = $1+(t / \mathrm{Myr}-1.77) / 0.01$. This gives Scenarios $1,2, \ldots, 9$ for $t=1.77,1.78, \ldots, 1.85$ Myr. Run set $\mathbf{B}$ which includes type I migration is denoted by an I subscript: e.g. Scenarios $1_{\mathrm{I}}, 2_{\mathrm{I}}, \ldots, 9_{\mathrm{I}}$. During these maturation runs the simulation inner edge was set at $0.1 \mathrm{AU}$ and any material passing interior to this boundary was eliminated and assumed to be consumed by the central star. The configuration of these matured solids disks at $t=1.77 \mathrm{Myr}$, the opening of the stranding window, are shown in Fig. 4 and Table 2 gives relevant data including values for the remaining total mass $M_{\text {solid }}$, the maximum protoplanetary mass $m_{\max }$, the numbers of surviving protoplanets and super-planetesimals $N$ and $N^{\prime}$, and the protoplanet to whole disk mass fraction $f_{\text {proto }}$. Since the scenarios of the present work start much closer together in time than those of our previous models (0.01 Myr vs. $0.15-0.5 \mathrm{Myr}$ ), the state of the solids disks generated for Scenarios 2-9 does not change greatly from that of Scenario 1.

It can be seen, when comparing with the initial condition data in Table 1, that planetary growth has been strong, especially where no type I migration is operating. In this case (the upper panel in Fig. 4), mergers have reduced protoplanets to a third of their former number, $m_{\max }$ is high (there being a $2.67 M_{\oplus}$ planet present at $2.01 \mathrm{AU})$, and $f_{\text {proto }}$ indicates that the accretion pattern of the disk has progressed way beyond oligarchic growth into the chaotic growth regime. Very little mass has been lost interior to $0.1 \mathrm{AU}(\sim 3 \%)$ via dynamical spreading and gas drag induced orbital decay of planetesimals. With type I migration, there is in play an additional preferential damping and inward migration of the most massive protoplanets (clearly visible in the lower panel in Fig. 4) resulting in the loss of $\sim 12 \%$ of the disk mass beyond the simulation inner edge. This loss is mostly in the form of large bodies as can be inferred from the lower values of $m_{\max }, N$, and $f_{\text {proto }}$. It might be thought that this loss is quite modest considering our inclusion of type I migration forces. However, in a rapidly dispersing gas disk model such as ours, inward type I migration, which is proportional to planetary mass, is limited at early times by the small size of protoplanets, and at late times by low gas densities (see also McNeil et al. 2005; Daisaka et al. 2006); Fig. 4 shows that at $t=1.77 \mathrm{Myr}$, $\Sigma_{\mathrm{g}}$ interior to $1 \mathrm{AU}$ has fallen by two orders of magnitude. The effect of type I migration on the radial distribution of solids disk mass is shown in Fig. 5 where the total solids mass for both models at $t=1.77 \mathrm{Myr}$ is plotted in 0.5 AU width bins against radial distance. It can be seen that beyond $\sim 2$ AU the radial mass profile of the disks in the two models remains similar, but interior to $2 \mathrm{AU}$ the more rapid pace of protoplanetary growth has resulted, in the type I migration case, in an inward displacement of mass caused mainly by a fractionation of the most massive bodies from the rest of the swarm which are now crowding the inner $0.5 \mathrm{AU}$ of the system. It was shown in Fogg \& Nelson (2007b) that hot-Earth type planets are more likely to accrete and survive when a giant planet migrates through such a solids disk, where previous type I migration has caused a radial contraction of the inner mass distribution.

The matured disks summarised above, aged in 10000 year stages from $t=1.77 \mathrm{Myr}$, are used as the basis for the type II giant planet migration scenarios presented here. In each case, a giant planet of $0.5 M_{J}$ is inserted at $5 \mathrm{AU}$ after removing $0.4 M_{J}$ of gas from the disk and the inner boundary of the simulation is reset to $0.014 \mathrm{AU} \cong 3 R_{\odot}$, which is approximately the radius of a solar mass T-Tauri star (Bertout 1989). The giant planet then proceeds to clear an annular gap in the gas and undergoes inward type II migration. The simulations are halted when the giant planet strands as a result of the near complete loss of the disk gas. In practise, since our viscous disk algorithm requires a finite amount of gas in each cell to remain stable, we assume that the giant halts inward migration when the migration rate falls below $-0.2 \mathrm{~cm} \mathrm{~s}^{-1} \cong-4.2 \times 10^{-7} \mathrm{AU} \mathrm{yr}^{-1}$. By this time the total gas remaining in the entire modeled disk is $<10^{-5} M_{\odot}$ and any error in stranding radius caused by this procedure is only on the order of $\sim 10^{-3} \mathrm{AU}$. The symplectic time-step for these runs was set to one tenth the orbital period of the innermost object which was achieved by dividing each simulation into a set of sequential sub-runs with the time-step adjusted appropriately at each restart. Since these new simulations involved a migration of roughly triple the simulated duration of our previous models (see Fig. 3), and since small time steps were usually needed during the long drawn out "end game" when the giant planet and the shepherded fraction of the solids disk are close to their final positions, these runs took a particularly long while to complete, requiring 4-6 months of $2.8 \mathrm{GHz}-\mathrm{CPU}$ time each.

\section{Results of the model}

\subsection{System configurations at the stranding point}

All scenarios, when run to the point at which the giant planet ceases migrating, exhibit a varying mix of the same shepherding and scattering effects on the solids disk shown in Fogg \& Nelson $(2005,2007 a, b)$. It is unnecessary therefore to repeat the previous procedure of giving a detailed account of the evolution 


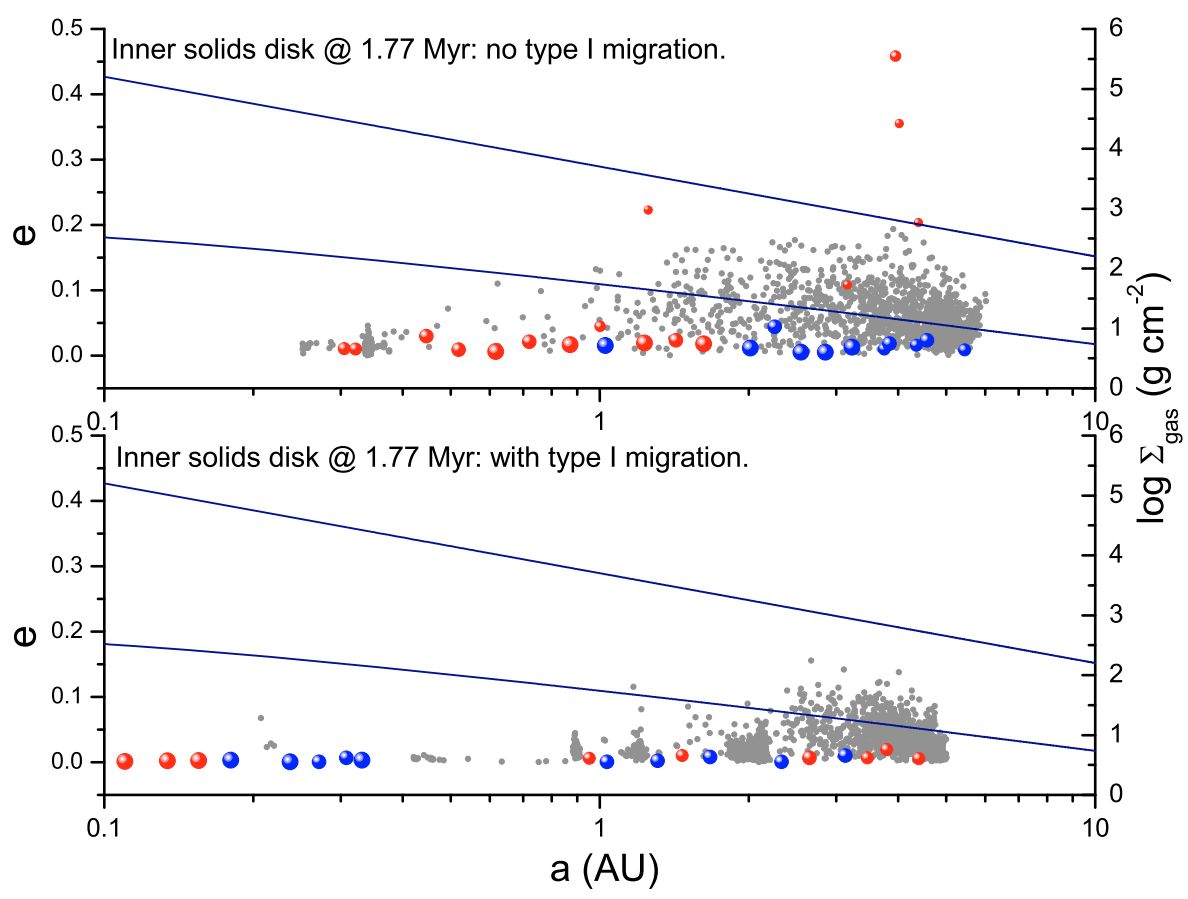

Fig. 4. Eccentricity vs. semi-major axis for matured solids disks at $1.77 \mathrm{Myr}$ for no type I migration (top panel) and with type I migration (bottom panel). Grey dots are superplanetesimals; red and blue circles are protoplanets originating interior and exterior to the snowline respectively. Gas densities are read on the right hand axes: the upper blue lines show gas density at $t=0$ and the lower cyan lines are the current densities.
Table 2. Matured solids disk at the start of the stranding window.

\begin{tabular}{c|cc}
\hline \hline$t$ Myr & 1.77 & 1.77 \\
Type I migration? & no & yes \\
\hline$M_{\text {solid }}$ & $33.52 M_{\oplus}$ & $30.42 M_{\oplus}$ \\
$m_{\max }$ & $2.67 M_{\oplus}$ & $1.62 M_{\oplus}$ \\
$N$ & 27 & 19 \\
$N^{\prime}$ & 1854 & 1809 \\
$f_{\text {proto }}$ & 0.62 & 0.52 \\
\hline
\end{tabular}

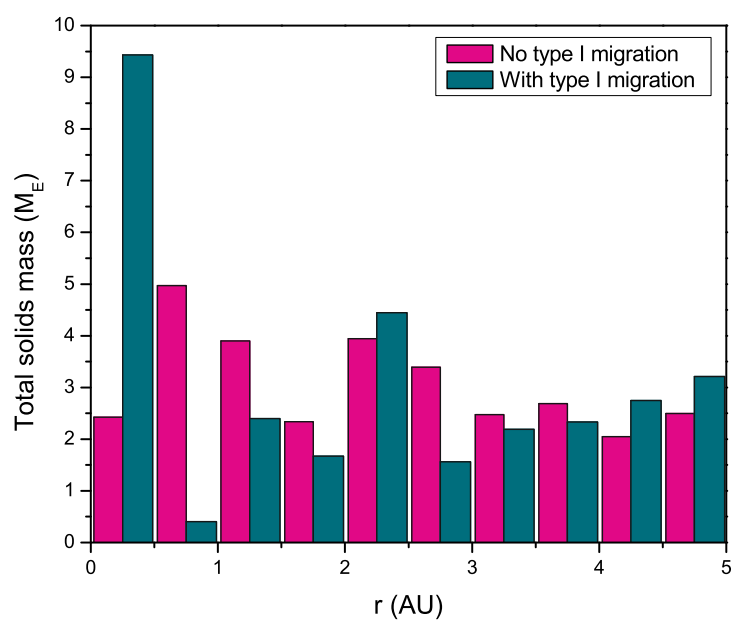

Fig. 5. Total solids mass in $0.5 \mathrm{AU}$ width bins at $t=1.77 \mathrm{Myr}$ for maturation runs with no type I migration (magenta bars) and with type I migration (cyan bars).

of one representative case. The big difference is that the scenarios presented here result in a more restricted type II migration, with the giant planet stranding between semi-major axes of $a_{\mathrm{g}} \approx 0.1-1.7 \mathrm{AU}$, in a context where all the damping forces that are dependent on the disk gas are close to their minimum possible values.
The end points ${ }^{2}$ of Scenarios 1-9, those without type I migration (Run Set A), are all illustrated in Fig. 6. Their counterparts, Scenarios $1_{\mathrm{I}}-9_{\mathrm{I}}$, with type I migration operating (Run Set B), are illustrated in Fig. 7. Comparison of the figures shows the familiar partitioning of the solids disk into shepherded interior and scattered exterior fractions, systematically truncated by the extent of the traverse of the giant planet. Early scenarios $\left(1-3\right.$ and $\left.1_{I}-3_{I}\right)$ are the closest to previous models and result in relatively better populated exterior disks and sparser interior disks without type I migration in force, and the opposite tendency with type I migration. This is in accord with the previous findings of Fogg \& Nelson (2007a) and Fogg \& Nelson (2007b) respectively.

No surviving protoplanets are found in or close to the system's maximum greenhouse habitable zone $(\sim 0.84-1.67 \mathrm{AU}$; Kasting et al. 1993) when the giant strands between $0.4 \lesssim a_{\mathrm{g}} \lesssim$ 1.2 AU. When stranding occurs at $a_{\mathrm{g}} \lesssim 0.4 \mathrm{AU}$, late scattered protoplanets can find themselves emplaced in the exterior disk at distances of $\$ 2 \mathrm{AU}$ and are hence candidates for evolving into future habitable planets. However, this eventuality appears less likely if type I migration is influential on the dynamics, as interior disk fractions evolve closer to the star, are better damped, and hence are less likely to lose their contents via late scattering. When stranding occurs at $a_{\mathrm{g}} \gtrsim 1.2 \mathrm{AU}$, protoplanets are found to survive in the inner regions of the $\mathrm{HZ}$ at $\sim 1 \mathrm{AU}$ in both scenario sets. The reason that interior $\mathrm{HZ}$ planets are found much closer to the giant planet than those in external orbits is simply a reflection of the asymmetry between shepherding and scattering behaviours. Shepherding of the interior population occurs between the $2: 1$ and $4: 3$ resonances, causing material to accumulate via disk compaction between $a \approx 0.63-0.83 a_{\mathrm{g}}$. In contrast, scattering typically results in the expulsion of a protoplanet into the exterior disk with an initial $e \gtrsim 0.5$ and periastron $\approx a_{\mathrm{g}}$ at the time of scattering; hence, exterior $\mathrm{HZ}$ planets are usually found with semi-major axes much larger than the final semi-major axis of the giant. Habitable planet candidates can therefore be

\footnotetext{
2 The phrase "end point" here refers to the time at which type II migration of the giant planet ceases due to gas disk dispersal.
} 


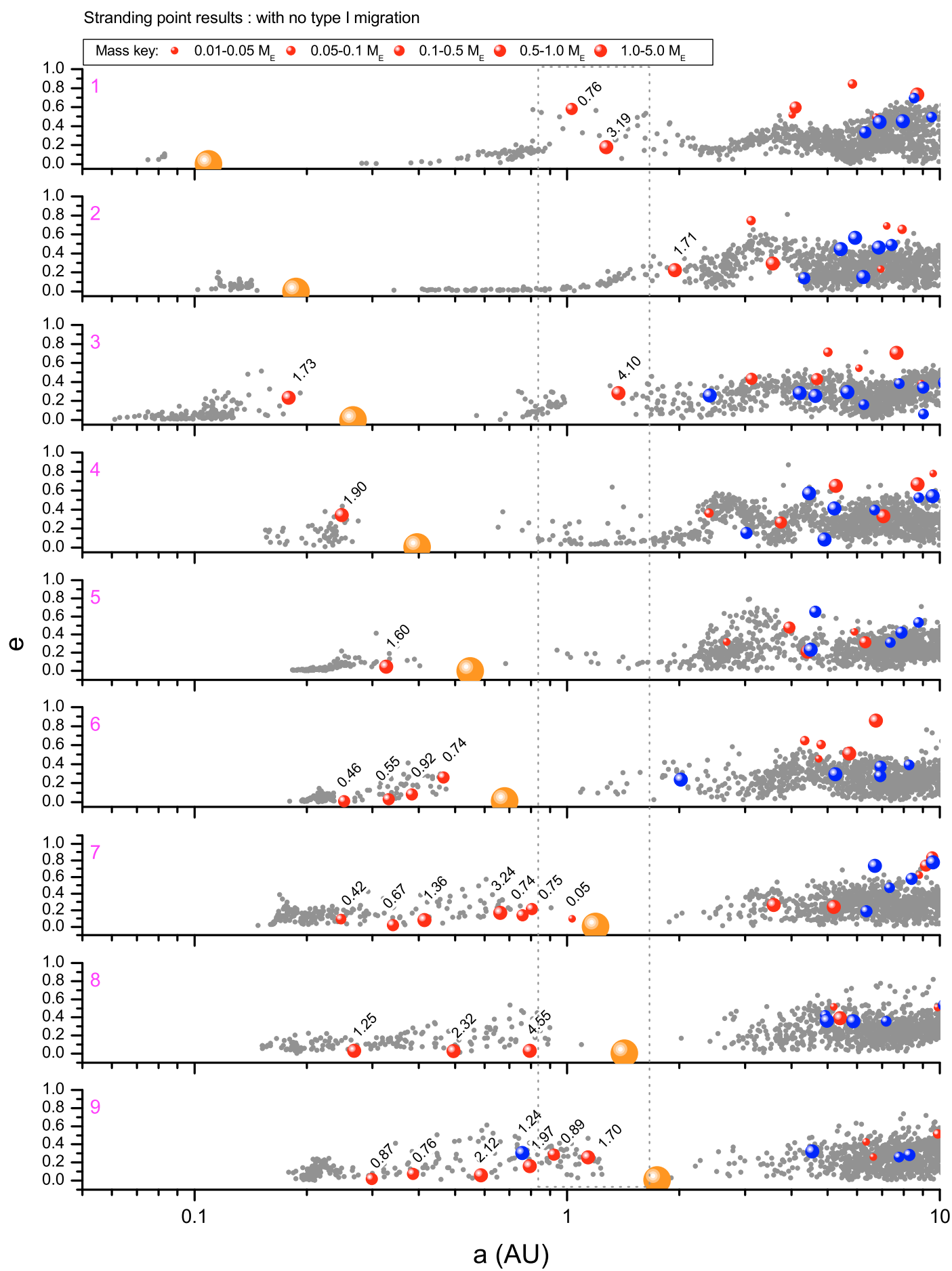

Fig. 6. Run Set A. End points of scenarios that exclude type I migration, when the giant planet strands at its final semi-major axis. Eccentricity is plotted vs. semi-major axis with symbols colour coded as in Fig. 4 and sized according to the mass key. Scenario ID is given at the top left of each panel. Protoplanets interior to the giant, or within 1-2 AU, are labelled with their mass in $M_{\oplus}$. The dotted box shows the habitable zone.

expected if a migrating giant planet makes a limited excursion into the HZ. However, if it traverses the HZ, such candidates are only expected to be common if the giant continues its migration to a radial distance of less than half that of the inner edge of the HZ. Whether the potential habitable planets visible in Figs. 6 and 7 can survive the long final phase of accretion that remains to be played out in their respective systems is examined in Sect. 3.2.

The interior disks that result after the giant planet's migration stalls show a considerable difference when the two figures are compared. When type I migration operates (Fig. 7), interior partitions tend to be more massive and have cleared almost all of their planetesimal population. Hence $f_{\text {proto }} \approx 1$, but large numbers of protoplanets are also found, as type I eccentricity damping exerted by the residual gas acts to reduce the effects of mutual scattering, causing protoplanets to dynamically settle into stable resonant convoys with closely spaced, and near-circular, orbits (originally described by McNeil et al. 2005). For example, the inner eight protoplanets at the end points of Scenarios $5 \mathrm{I}-7_{\mathrm{I}}$, from the inside out, are locked into a 4:3, 5:4, 4:3, 3:2, 5:4, 


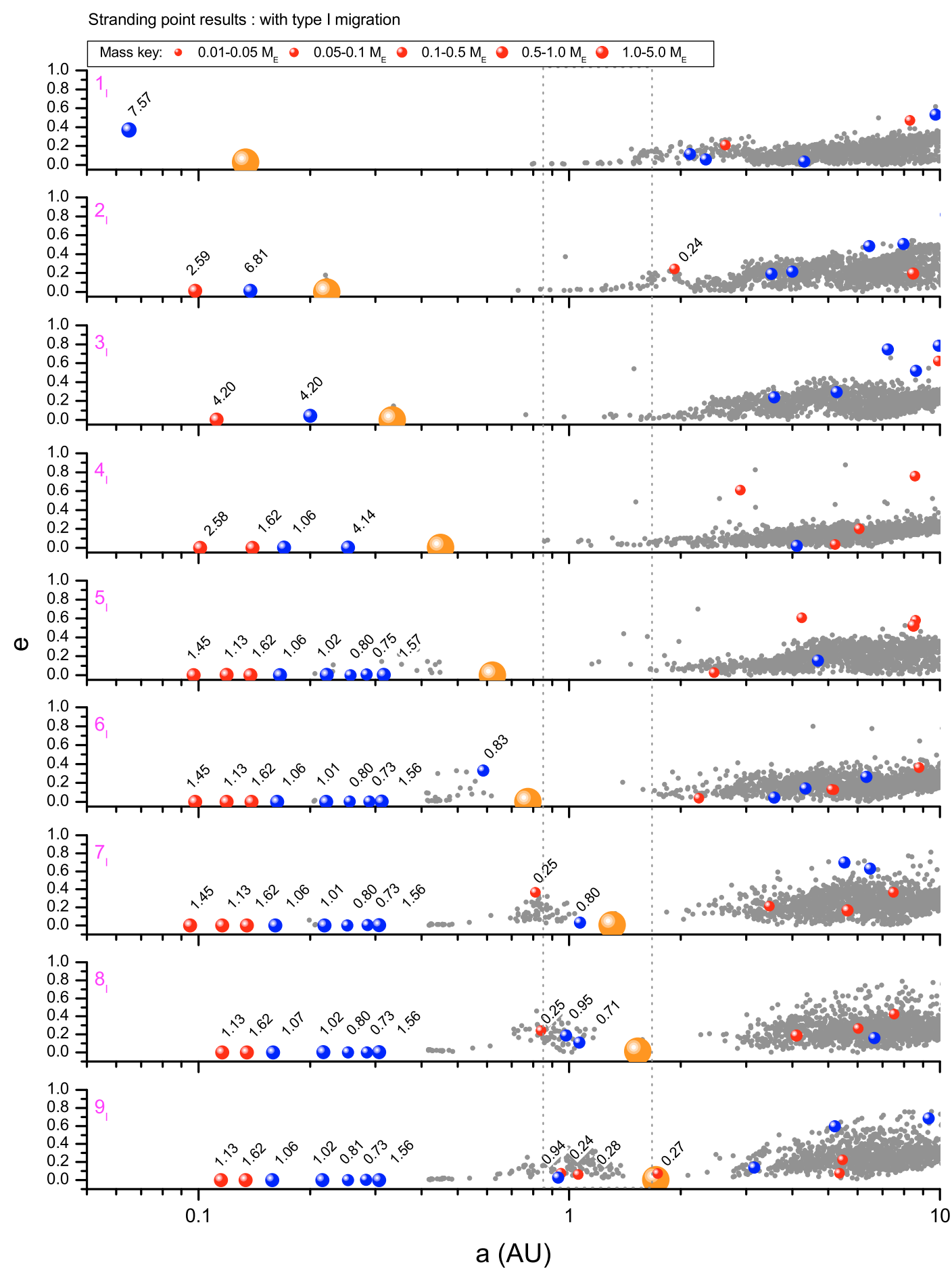

Fig. 7. Run Set B. End points of scenarios that include type I migration, when the giant planet strands at its final semi-major axis. Eccentricity is plotted vs. semi-major axis with symbols colour coded as in Fig. 4 and sized according to the mass key. Scenario ID is given at the top left of each panel. Protoplanets interior to the giant, or within 1-2 AU, are labelled with their mass in $M_{\oplus}$. The dotted box shows the habitable zone.

7:6, 7:6 configuration of mean motion resonances. Oligarchic growth therefore ends in these interior systems when the planetesimal field is accreted, but giant impact growth is delayed, at least for as long as the disk gas persists. When no type I migration operates (Fig. 6), interior partitions are relatively depleted of mass and contain fewer protoplanets, in more excited orbits, alongside a surviving population of planetesimals $\left(f_{\text {proto }}<1\right)$. In Scenarios 1-2, where the giant planet has experienced the lengthiest migration to $a_{\mathrm{g}} \lesssim 0.2 \mathrm{AU}$, late scattering or accretion has removed all interior protoplanets (similar to the results of Fogg \& Nelson 2007a). In Scenarios 3-5, just one relatively low mass hot-Earth remains at the end point. The inner disk fares better in late scenarios (7-9) when migration is limited to $a_{\mathrm{g}} \gtrsim 1 \mathrm{AU}$. Numerous interior protoplanets remain in these cases, and since type I migration forces are absent, accretion via giant impacts is not suppressed and protoplanetary growth is more advanced. Since both run sets produce such different interior partitions, it is of considerable interest to determine if this effects their final architectures after the subsequent phase of gasfree accretion. This issue is followed up in Sect. 3.2. 
Some features of the interior systems illustrated in Figs. 6 and 7 are worthy of further comment.

Scenarios 3-5: only one interior planet survives in each of these scenarios where the giant planet strands between $a_{\mathrm{g}}=$ $0.27-0.55 \mathrm{AU}$, and only one of these is found at a first order resonance with the giant at the end point. This is the $1.90 M_{\oplus}$ planet in Scenario 4, which is captured at the 2:1 resonance. In the case of Scenario 3, the interior planet is found closer to the giant planet, whilst in Scenario 5 the sweeping 2:1 resonance has not quite reached the surviving $1.60 M_{\oplus}$ planet.

Scenario 6: the giant planet has stranded at $a_{\mathrm{g}}=0.68 \mathrm{AU}$, leaving four surviving protoplanets in the interior partition. The outermost of these has an orbit with $e \approx 0.3$, close to a 7:4 period ratio with the giant planet, that crosses the orbit of its nearest neighbor. To an accuracy of $<1 \%$, period ratios between the protoplanets, from the outside in, are 4:3, 5:4, and 3:2 respectively, which are a feature reminiscent of the resonant convoys of protoplanets commonly observed in simulations where type I eccentricity damping is included. In this case, dynamical friction from surviving planetesimals exerts the damping, but its relative weakness produces an arrangement that is more dynamically excited and clearly unstable.

Scenario 8: this case stands out from its adjacent Scenarios 7 and 9, where the giant planet also strands at $a_{\mathrm{g}}>1 \mathrm{AU}$, because planetary growth in the interior partition appears to be much more advanced, resulting in three planets in well-spaced orbits. This is entirely due to chance giant impacts shortly before the scenario end point. It is shown in Sect. 3.2 that, if carried through into the gas-free phase, accretion within the interior partitions of Scenarios 7 and 9 rapidly catches up, with excess protoplanets being eliminated by co-accretion or impact onto the giant planet.

Scenario $1_{\mathrm{I}}$ : the single interior planet resulting from this run is the best hot-Neptune/super-Earth analogue generated in this paper. Its mass of $7.57 M_{\oplus}$ puts it within the observed range for such objects. However, in contrast with the results of Fogg \& Nelson (2007b), the planet is not found at the first order 3:2 or 2:1 resonances, but it is located further away from the giant planet, at the second order 3:1 resonance (confirmed with a plot of librating resonant angles). Study of this system's evolution however reveals that this object was originally shepherded inward at the $2: 1$, but within the last $0.12 \mathrm{Myr}$ of the run it scatters with and eventually accretes two other protoplanets interior to it, contracting the orbit of the merged body, whereupon it is fortuitously captured at the 3:1 resonance. This phenomenon of resonance capture through scattering has been recently described by Raymond et al. (2008). The arrangement of hot-Neptune and hot-Jupiter in Scenario $1_{\mathrm{I}}$ is most similar to that observed between the two inner planets $e$ and $b$ in the 55 Cancri system (McArthur et al. 2004), although the orbits of these two natural objects are separated by a wider $\sim 5: 1$ period ratio.

Scenario 2I: two interior planets survive in this case where the giant strands at $a_{\mathrm{g}}=0.22 \mathrm{AU}$. The outermost planet of this pair is in the 2:1 resonance with the giant planet.

Scenario $3 \mathrm{I}$ : two equal mass hot-Earths survive at the end point of this scenario $\left(a_{\mathrm{g}}=0.33 \mathrm{AU}\right)$, but neither of them are in resonant orbits. A resonant convoy of five interior planets becomes unstable in the last $40000 \mathrm{yr}$ of the simulation and is broken up by giant impacts.

Scenario $9 \mathrm{I}$ : a noteworthy feature of the end point of this run is the presence of a $0.27 M_{\oplus}$ Trojan protoplanet, located in a stable co-orbital resonance with the giant planet. This object was captured into this 1:1 resonance shortly after the introduction of the giant and escorts the larger body inward during

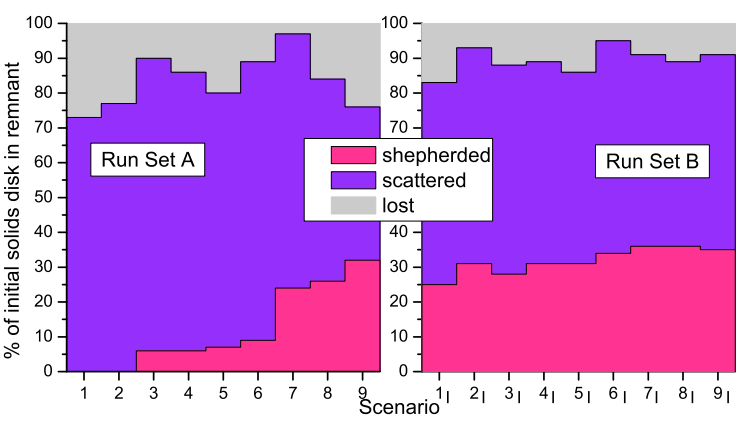

Fig. 8. Fate of the disk mass at the end points of Run Set $\mathbf{A}$ and $\mathbf{B}$. Data include the cumulative \% of initial solids disk in the shepherded or scattered remnant, or lost from the disk.

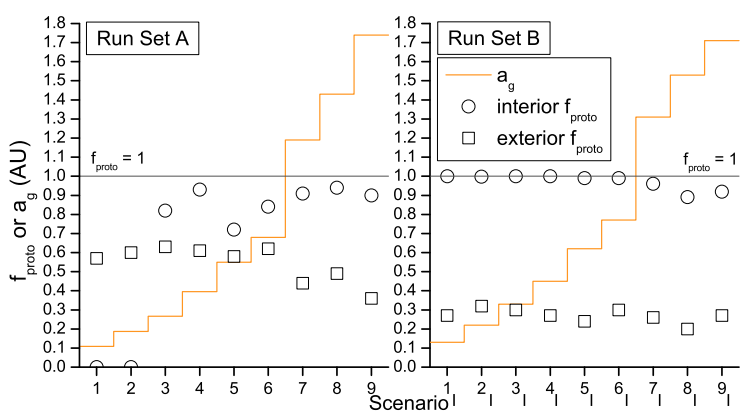

Fig. 9. Protoplanet mass fraction $f_{\text {proto }}$ interior to giant (circles) and exterior to giant (squares). Also shown is the stranded giant planet semimajor axis $a_{\mathrm{g}}$ (stepped line) at the end points of both Run Sets.

type II migration. Although the capture of the Trojan planet in this case may have been influenced by the abrupt introduction of the giant planet at the scenario start time, its presence at the end point is not necessarily unrealistic or unexpected. Dvorak et al. (2004) and Erdi \& Sandor (2004) have shown that co-orbital motions can be stable for long time scales and 1:1 capture and co-migration, in a context where type I migration forces are active, has previously been observed in simulations by Cresswell \& Nelson $(2006,2008)$.

Alternate visualizations of the data for the end points of Scenarios 1-9 and Scenarios $1_{\mathrm{I}}-9_{\mathrm{I}}$ are presented in Figs. 8 and 9 respectively. These data are the equivalent of the "fate of the solids disk mass" results of previous models set out in Tables in the previous papers in this series and have to be recast into graphical format here since 18 scenarios are under consideration, rather than 5 or 6 . The nine scenarios of each run set are indicated on the $x$-axis with data from Run Sets $\mathbf{A}$ and $\mathbf{B}$ being shown in the left and right hand panels respectively. In Fig. 8, the cumulative percentage of the initial solids disk remaining is read off the $y$-axis, with the red bars representing the fraction remaining in the shepherded remnant, the blue bars that in the scattered remnant, and the grey bars that which is lost - predominantly via accretion onto the giant planet. In Fig. 9, the $y$-axis gives both $f_{\text {proto }}$ for the interior or exterior remnant (the symbols being given in the key), and the stranding position of the giant planet $a_{\mathrm{g}} / \mathrm{AU}$ which is shown by the orange line.

Figure 8 shows that the great majority of the solids disk ( $\sim 80-90 \%)$ survives giant planet migration, regardless of whether type I migration operates or not, and regardless of the stranding position of the giant planet. The bias toward scattering behaviour in the absence of type I migration shows well in the data for Run Set $\mathbf{A}$ as, when $a_{\mathrm{g}}<1 \mathrm{AU}$, less than $10 \%$ of the disk mass remains in the shepherded partition. The biassed 
scattering of protoplanets is indicated by the value of exterior $f_{\text {proto }}>0.5$. In contrast, in the presence of type I migration (Run Set B), partitioning of the solids disk is largely insensitive to $a_{\mathrm{g}}$ with $\sim 30 \%$ and $\sim 60 \%$ of the mass remaining in the interior and exterior partitions respectively in all scenarios. The bias against the scattering of protoplanets is indicated by the value of exterior $f_{\text {proto }} \approx 0.3$.

One might reasonably speculate, given the details in Figs. 6-9, that the final systems of terrestrial planets that should emerge from these scenarios might be either internally or externally weighted (in terms of both planetary numbers and masses) depending on the strength of type I migration forces operating during gas-phase formation. If the model reflects reality, its results may help contribute to the debate over the reality of type I migration once observational techniques have advanced sufficiently to enable a more complete inventory of terrestrial planets in exoplanetary systems where type II giant planet migration is thought to have occurred.

\subsection{Post-migration terrestrial planet formation}

The results of the model, shown in Figs. 6 and 7, have taken the accretion process only to the point at which the giant planet strands due to the loss of the nebular gas. Planet formation in all exterior and most interior partitions of the original inner system disk is clearly incomplete and awaits a much lengthier phase of gas-free accumulation. Simulation of at least part of this gas-free accretion phase is of clear interest to see if the conclusions drawn from running the model to the end point of migration might stand up over the long-term. Would a more complete accretion reduce the observed differences between the two run sets? What will be the fate of the closely packed resonant convoys of protoplanets created by type I migration (Fig. 7)? Are habitable zones containing protoplanets at the end of the migration epoch still likely to contain planets over the long-term?

Thus, after switching off all residual forces exerted by the last traces of gas, simulation of all the scenarios of both Run Sets has been extended with the aim of running them to a system age of $30 \mathrm{Myr}(t=29.5 \mathrm{Myr})$. However, this is not practical in every case as the time-step is constrained by the orbits of the innermost objects. The method adopted here is to choose a time step equivalent to one tenth of a circular orbit with a semi-major axis equal to the periastron of the innermost large object. This strategy typically dictates a time-step of 2-4 days and serves well in all but two scenarios. These are Scenario 3 in which a 2 day time-step inadequately resolves the orbits of a planetesimal swarm interior to the hot-Earth at $0.18 \mathrm{AU}$, resulting in an unrealistic loss of these objects via accretion onto both the star and the hot-Earth; and Scenario $1_{\text {I }}$ where a hot-Neptune at $0.065 \mathrm{AU}$ dictates too small a time-step - a problem that is tackled by splitting the problem into sub runs -1 ) the hot-Neptune and hot-Jupiter with a 0.3 day time-step; 2) the hot-Jupiter and external disk with a 2 day time-step, and then assembling both as a composite. Even so, eight months after beginning most of these extended runs it has not been possible before submission of this paper to complete every simulation. All scenarios in Run Set $\mathbf{A}$ have attained the goal of $30 \mathrm{Myr}$, but in Run Set $\mathbf{B}$, where previous type I migration has typically resulted in protoplanets closer to the star at the point of gas loss, the results presented here vary in their ages from 20-30 Myr.

The results of these extended simulations are illustrated in Figs. 10 (Run Set A) and 11 (Run Set B) and should be compared with their migration end-point equivalents in Figs. 6 and 7 respectively. A number of general observations can be made.
1. Accretion interior to the giant planet. Solitary, or paired, hotNeptunes or hot-Earths that are present at the end of the migration epoch (e.g. Scenarios 3-5 and Scenarios $1_{I}-3_{I}$ ) are found to survive the extended gas-free accretion phase with little change in their orbital parameters. The crowded interior disk partitions that result in later scenarios where the giant comes to rest at greater distances from the central star undergo rapid evolution to a state of near completion, with giant impacts thinning down the number of protoplanets which mop up almost all residual planetesimal debris.

As discussed in Sect. 3.1, giant impact growth in Run Set A is already underway before the end of the migration phase as the protoplanets within interior partitions are sufficiently dynamically excited to exhibit crossing orbits. This process completes rapidly after gas loss, with protoplanetary mergers reducing their numbers by $\sim 50 \%$, resulting in apparently stable multiple interior systems of two or three hot-Earths separated by $>20$ mutual Hill radii (note Scenarios 6-9, Fig. 10). In Run Set $\mathbf{B}$, type I migration forces have previously suppressed giant impact growth, causing protoplanets to stack into crowded resonant convoys (e.g. Scenarios $5_{\mathrm{I}}-9_{\mathrm{I}}$, Fig. 7). After the gas is gone in these cases, all eccentricity damping ceases and the convoys start to destabilize within a few Myr, typically by protoplanets merging with their nearest neighbours, ultimately reducing their numbers by $\sim 50 \%$. This occurs fastest in convoys that are the most compressed by the position of the giant planet (i.e. restricted within the narrowest annulus) and the process is clearly illustrated in the case of Scenario 5I in Fig. 12. Generally however, systems where strong type I migration has operated in the gas phase still retain more interior planets, although is it not certain that all giant impact growth is completed within the interior partitions of the later scenarios illustrated in Fig. 11. Scenarios 7 I and 9 I have interior protoplanets still separated by as little as $\sim 10$ mutual Hill radii and some traces of the original resonant convoy structure remains, such as the $4: 3$, $5: 4,4: 3$ commensurability between the inner four planets of Scenario $7_{\mathrm{I}}$. To test the stability of the still crowded inner systems shown in Fig. 11, the orbits of the giant and the remaining interior planets in Scenarios $7_{\text {I }}$ and 9 I $_{\text {I }}$ were integrated to $t=100 \mathrm{Myr}$ and the results are displayed in Fig. 13. It can be seen that whilst the inner system of seven planets in Scenario 9 I is stable over this extended time frame (including the orbit of the Trojan planet), that of Scenario 7 I undergoes further orbital evolution and accretion resulting in a system of four survivors. Whatever the final outcome in these cases however, hot and warm-Earths, including multiple systems, are predicted as an outcome of type II migration induced disk compaction in all but the earliest scenarios of Run Set $\mathbf{A}$.

2. Accretion exterior to the giant planet. Planetary growth in exterior partitions at $\gtrsim 2 \mathrm{AU}$ is ongoing and not yet complete. This is to be expected as dynamical times are longer, scattered material is spread over a larger volume, and does not have an opportunity to interact with disk solids originating beyond $5 \mathrm{AU}$ which have not been modelled. However, it can be seen in Figs. 10 and 11 that dynamical friction exerted by the scattered planetesimal population has resulted in a degree of circularization of the orbits of scattered protoplanets, especially in the case of Run Set $\mathbf{B}$, where dynamical friction is particularly strong due to the low exterior value of $f_{\text {proto }}$ (see Fig. 9 and associated discussion).

3. Planetary occupants of the habitable zone. Three planets in each run set are found in their system's habitable zone ( 0.84-1.67 AU) after the extended gas-free runs. In 


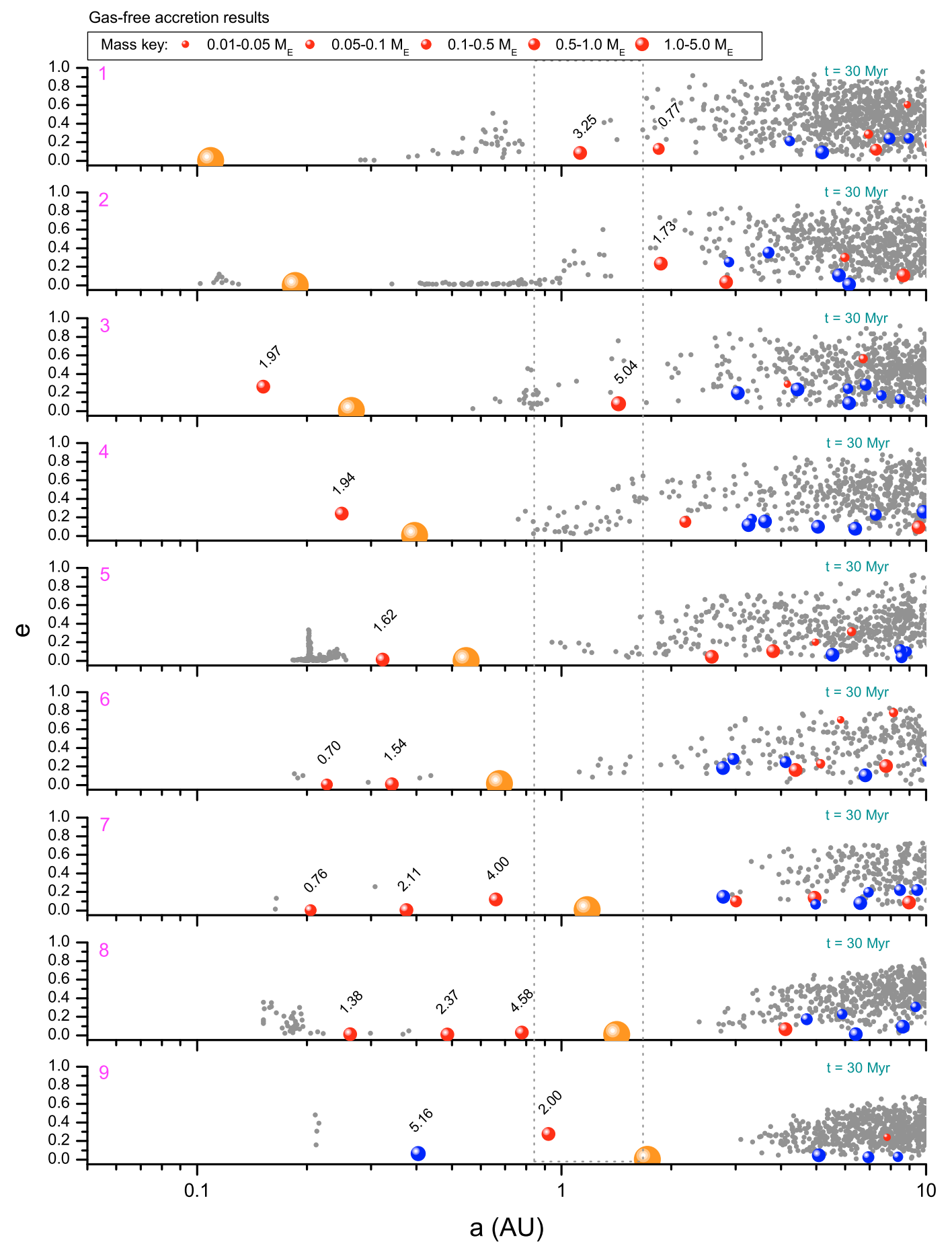

Fig. 10. Run Set A. Gas-free accretion results of scenarios that exclude previous type I migration. Eccentricity is plotted vs. semi-major axis with symbols colour coded as in previous examples and sized according to the mass key. Scenario ID is given at the top left of each panel. System age is given at the top right of each panel. Protoplanets interior to the giant, or within $1-2 \mathrm{AU}$, are labelled with their mass in $M_{\oplus}$. The dotted box shows the habitable zone.

Run Set $\mathbf{A}$, these are 3.25, 5.04 and $2.00 M_{\oplus}$ planets in Scenarios 1, 3 and 9 respectively (Fig. 10); and in Run Set $\mathbf{B}$ a $1.66 M_{\oplus}$ planet in Scenario $8_{\mathrm{I}}$ and 1.26 and $0.27 M_{\oplus}$ planets in Scenario 9 I (Fig. 11). This latter object is the Trojan planet captured into a 1:1 resonance with the giant early on in the migration phase (discussed in Sect. 3.1) and dragged inward to $a=1.66 \mathrm{AU}$, just inside the outer edge of the habitable zone. This exotic world remains in a continuously stable orbit up to a system age of $30 \mathrm{Myr}$, and has the potential for a prolonged existence (Dvorak et al. 2004; Erdi \& Sandor 2004, and Fig. 13). The finding articulated in the previous Section that the giant planet must make either a limited excursion into the habitable zone, or a complete traversal down to $\lesssim 0.5 \times$ the radial distance of the inner edge of the HZ, for a habitable planet to be possible is reinforced by the results of the extended simulations. Potentially habitable planets are found in systems where the giant planet lies outside 


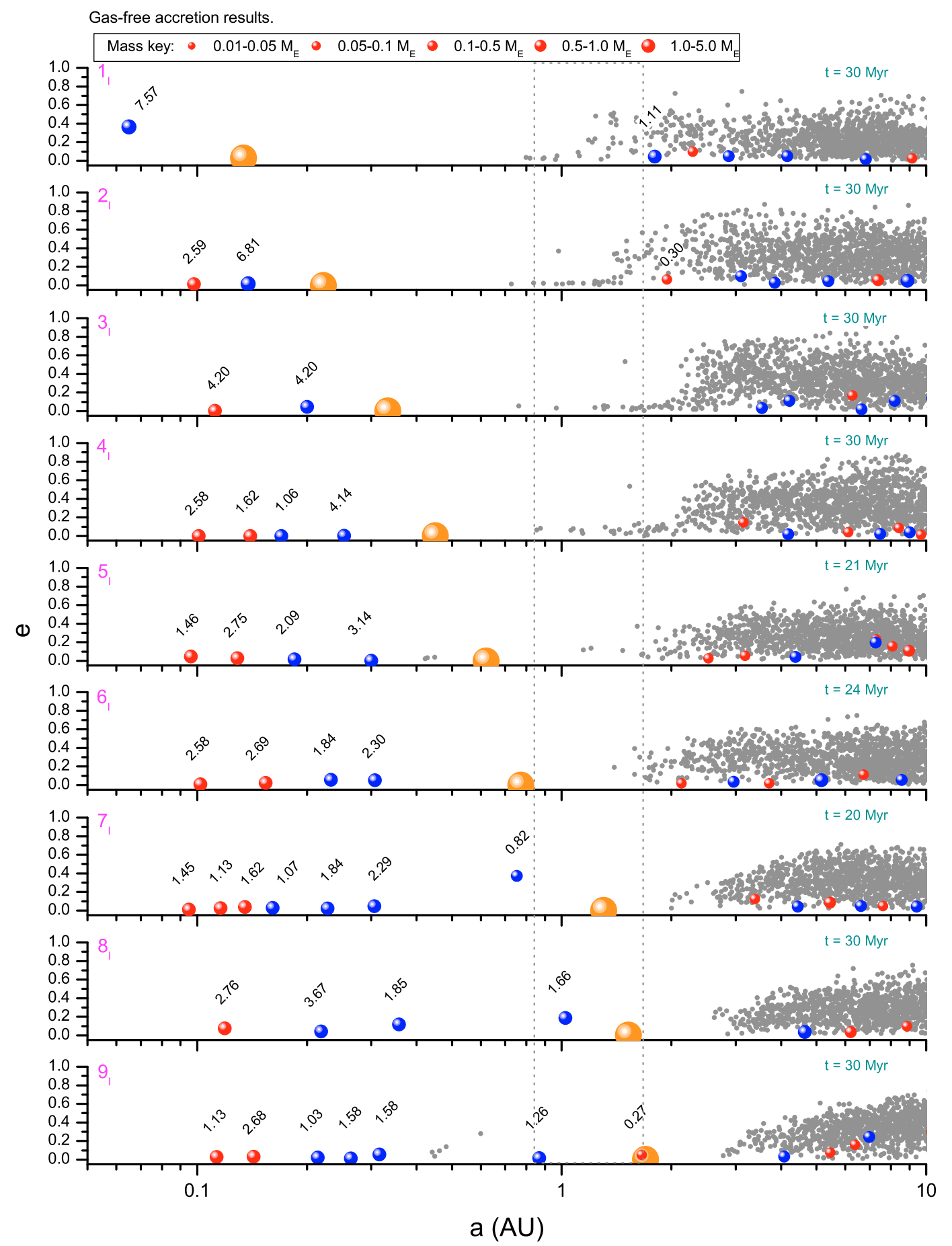

Fig. 11. Run Set B. Gas-free accretion results of scenarios that include previous type I migration. Scenario ID is given at the top left of each panel. System age is given at the top right of each panel. Protoplanets interior to the giant, or within 1-2 AU, are labelled with their mass in $M_{\oplus}$. The dotted box shows the habitable zone.

the region of $\sim 0.3-1.5 \mathrm{AU}$, except in the case where a Trojan planet accompanies a giant stranded within the $\mathrm{HZ}$.

4. Volatile endowments. In agreement with previous models (Fogg \& Nelson 2007a,b) and the work of Raymond et al. (2006) and Mandell et al. (2007), the habitable planets generated in the present model are predicted to be richly endowed with volatiles driven inward from beyond the nebular snowline by the migrating giant planet. The composition of the six habitable planet candidates, using the same crude three phases described in Fogg \& Nelson (2007a), are shown as pie charts in Fig. 14 where the red sectors refer to dry material originating interior to $2 \mathrm{AU}$, the green sectors to chondritic material originating between 2 and $2.7 \mathrm{AU}$, and the blue sectors indicate the fractions originating from beyond the snowline at $>2.7 \mathrm{AU}$. Even with no previous type I migration operating (Run Set A), the habitable planet candidates mentioned above have incorporated roughly a third to a half of their material from beyond the snowline. Where there has been pre-existing type I migration (Run Set B), more like three quarters of the planets' mass originates from beyond 


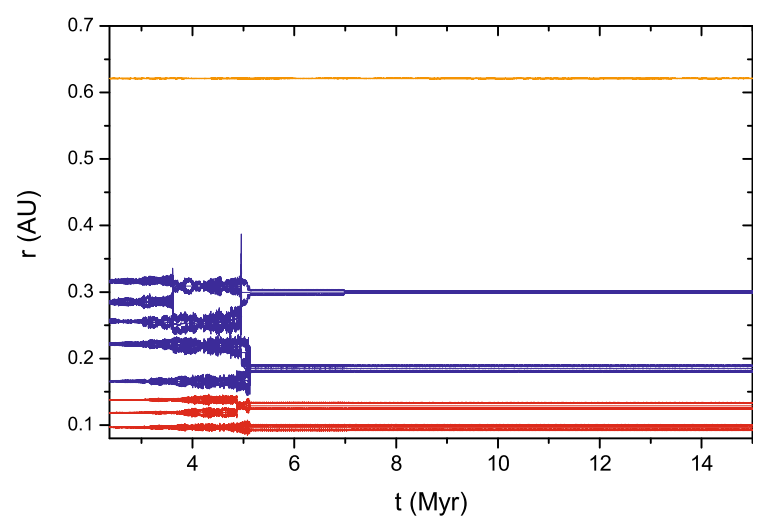

Fig. 12. Destabilization of the resonant convoy in Scenario 5. Temporal evolution of the periastron, semi-major axis, and apastron for each object is shown, with the giant planet drawn in orange at the top of the graph. Protoplanets undergo a sequence of closest neighbour mergers, reducing their number from eight down to a stable quartet of hot-Earths.

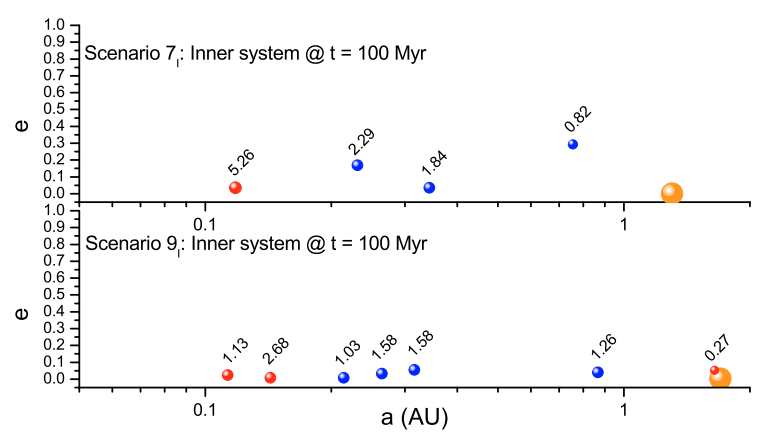

Fig. 13. Inner systems of Scenarios $7_{\mathrm{I}}$ and $9_{\mathrm{I}}$ run to $t=100 \mathrm{Myr}$.

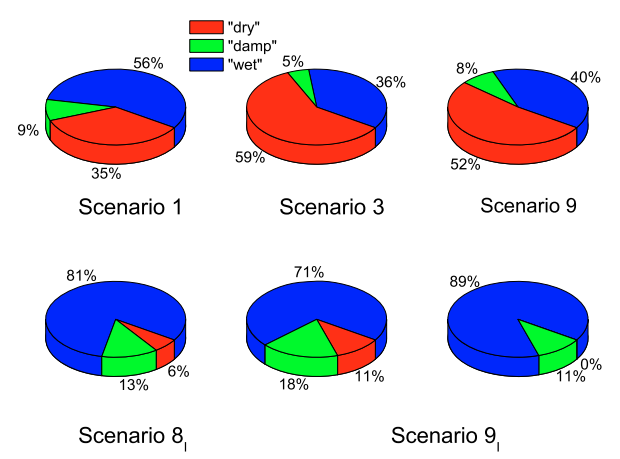

Fig. 14. Material composition of habitable planet candidates from our simulations. Red indicates material of provenance $a<2 \mathrm{AU}$; green indicates material of provenance $2 \mathrm{AU}<a<2.7 \mathrm{AU}$; and blue indicates trans-snowline material originating from $a>2.7 \mathrm{AU}$. The top row of charts are the results of Run Set $\mathbf{A}$ and the bottom charts the results of Run Set B.

the snowline. Whether the habitable planets in question lie interior or exterior to the giant seems to make little difference to the composition. All such worlds are predicted to be richly endowed with water and gases.

\section{Discussion}

The key finding of the model presented in this paper, and its predecessors (Fogg \& Nelson 2005, 2006, 2007a,b), that giant planets do not remove the portion of a protoplanetary disk they migrate through, or prevent subsequent terrestrial planet formation, is robust in the sense that every model variant, and independent work (Raymond et al. 2006; Mandell et al. 2007), has reproduced it. However, these models have only explored a modest region of parameter space relevant to the problem, and have inevitably adopted assumptions that simplify or omit potentially important physical processes. We have discussed a number of issues concerning parameter variations and neglected physical processes in our previous papers and restrict the discussion here to three caveats not previously considered.

i) Solids disk inner boundary. The inner system solids disk adopted here begins with its inner boundary set at 0.4 AU. Its contents are then free to evolve inward during the maturation runs preceding the introduction of the giant at the start of a migration scenario. By this time, in low dissipation models (Run Set A), dynamical spreading typically results in a modest inward movement of the disk edge to $\sim 0.3 \mathrm{AU}$ (see the upper panel of Fig. 4) so that when the giant planet subsequently migrates down to $0.1 \mathrm{AU}$ it passes through the entire inner disk annulus. This is not necessarily the case in high dissipation models (Run Set B) where greater inward movement of the solids disk contents occurs during the maturation phase (see the lower panel of Fig. 4). Thus, the question is raised as to whether the lack of interior hotEarths resulting in Scenarios 1 and 2 is a realistic finding or an artifact of the initial position of the solids disk inner boundary. What if this boundary had been set at $0.1 \mathrm{AU}$ instead? This would have provided an additional $\sim 3 M_{\oplus}$ of material from which potential interior planets could have been assembled. It cannot be ruled out therefore that some hot-Earths could grow and survive even in early scenarios of Run Set A, especially if the giant planet stops short of their position. However, one would still expect them to be of lower mass and more prone to orbital destabilization than in high dissipation models where eccentricity damping is in play and where there has been much more extensive prior inward migration of solids.

ii) Random component of initial conditions. The solids disk with which we begin our simulations at $t=0$ conforms as a whole to a $3 \times$ MMSN model, but is unique in the sense that its individual particles start with randomized orbital elements. This raises the question of how the conclusions of this paper might depend on the seed number used to randomize these initial conditions. We have not tested the influence of alternate seed numbers on the particular set of simulations presented here. However, comparison with our previous work, where different integers have been used to seed the initial setup, shows that in scenarios where disk maturity and dissipative forces are roughly equivalent, the overall architectures of the post-migration systems are similar even though details of individual planets are different. This is because the general physical processes shown to characterise the migration of a giant planet through an inner system disk, which lead to the division of the original disk contents into internal and external remnants, are largely independent of the random component in the initial conditions and are influenced primarily by the level and variety of dissipative forces in play - i.e. by the maturity of the solid body swarm, the density of the gas, and whether type I migration is active. An example of such a comparison can be seen by referring to Fig. 12 from Fogg \& Nelson (2007a), where the results of a late scenario, matured for $1.5 \mathrm{Myr}$ before the onset of giant planet migration, in the absence of type I migration, are plotted. The lower panel of this figure shows the evolution of this model 2 Myr after the giant planet has stranded at $0.1 \mathrm{AU}$ 
and its overall system architecture is similar to its closest equivalent in the present work: Scenario 1, shown in the top panel of Fig. 6. No interior hot-Earth is present and protoplanets amounting to several $M_{\oplus}$ have been externally scattered to between 1-3 AU in both cases. The post migration evolution of this hot-Jupiter system from Fogg \& Nelson (2007a) has been run further to $30 \mathrm{Myr}$ and the results illustrated in Fogg (2008). The resemblance between that run and the matured Scenario 1 in this paper (Fig. 10, top panel) is even closer with a $\sim 3.3 M_{\oplus}$ planet being present in the habitable zone of both systems. We conclude that although the specific properties of the planetary systems which arise from our simulations depend on the exact details of the initial setup, the general trends that we observe in our outcomes are robust.

iii) Ocean planets. One effect of giant planet migration is to drive large quantities of icy material into the inner system, with the outcome that terrestrial planets that form in the aftermath are predicted to be richly endowed with volatiles and enveloped by global oceans. There is nothing about $100 \%$ ocean cover that necessarily rules out the presence of life. Indeed, life may have started in the Earth's oceans, and exploration beneath ocean-covered Europa's ice shell has long been regarded as a top priority by astrobiologists (Reynolds et al. 1983, 1987). Ocean planets in the $\sim M_{\oplus}$ range however have a problem not shared with Europa: if the depth of their oceans is $\gtrsim 100 \mathrm{~km}$, their floors are composed of high pressure phases of ice, rather than rock, with the ice-silicate interface (and much potentially crucial chemistry) sealed off beneath an icy mantle $100 \mathrm{~s}$ or $1000 \mathrm{~s}$ of $\mathrm{km}$ thick, depending on the planet's total mass and bulk water content (Léger et al. 2004). How this would effect the solute content of the oceans is unknown but one might speculate that some trace elements essential for terrestrial life might be lacking ${ }^{3}$. Meteoritic infall after planetary differentiation is complete would re-supply some rock-forming elements for dissolution into the ocean, but solid material that deposits on the ocean floor would be denser than the ice beneath and prone to removal by sinking into the mantle. A related problem deepens this uncertainty. Planets overendowed with water might similarly possess massive atmospheres with unexpected compositions and properties. If ocean planets are more like thawed out versions of Titan, rather than water-rich Earths, then habitable zone calculations that rely on models of modest $\mathrm{CO}_{2} / \mathrm{H}_{2} \mathrm{O}$ atmospheres, coupled to a carbonate-silicate cycle and continental weathering (e.g. Kasting et al. 1993; Selsis et al. 2007; von Bloh et al. 2007), may not be relevant.

If the water content of trans-snowline planetary building blocks is assumed to be $\sim 75 \%\left(\sim 1-f_{\text {ice }}^{-1}\right.$ where $f_{\text {ice }}=4.2$ is the factor in the MMSN model by which nebular condensate masses are enhanced due to ice condensation), then the models presented here predict $\mathrm{HZ}$ planets composed of $\sim 20 \%-60 \%$ water. This is a vast inventory compared with the Earth's $\sim 0.1 \%$ water. It is likely though that these figures are overestimated. If the snowline is further from the star than the 2.7 AU assumed in the MMSN Model, or if the giant planet forms closer to it, the material shepherded inward by type II migration will have a lower volatile content. In the models of Raymond et al. (2006) and Mandell et al. (2007), where the snowline is placed at 5 AU, $\mathrm{HZ}$ planets are produced with a water content of $\sim 10 \%-\mathrm{a}$

3 The elemental requirements of non-terrestrial life are another matter and unknown. reduction from the above estimate, but still a factor of 20 greater than the typical outcome of $\sim 0.5 \%$ water content when models are run without a migrating giant planet (Raymond et al. 2004, 2007). However, all these models almost certainly overestimate the quantity of water retained by growing planets since loss of volatiles during accretion is not accounted for. Extensive depletion of both atmosphere and ocean could result from giant impacts late in formation (Genda \& Abe 2005; Asphaug et al. 2006; Canup \& Pierazzo 2006) which could strip nascent ocean planets down to a more Earth-like remnant. All of the HZ planets generated by the models presented here undergo at least one giant impact at some point in their evolution, with a minority suffering a high velocity collision with a comparable sized object during the later stage of accretion within the scattered disk. Thus, whilst ocean planets in habitable zones are a robust prediction of the model, it is premature to say that they would invariably occur in nature and would inevitably be uninhabitable.

\section{Conclusions}

All previous published models of terrestrial planet formation in the presence of type II giant planet migration have neglected the issue of what causes the giant planet to come to rest in its final orbit and have simply switched off migration forces at a pre-defined distance from the central star (Fogg \& Nelson 2005, 2007a,b; Raymond et al. 2006; Mandell et al. 2007). This latest model is the first simulation of this type to include a selfconsistent scenario for the stranding of the giant planet, which comes to rest naturally at the point when the gaseous fraction of the protoplanetary disk is lost via a combination of accretion onto the central star and photoevaporation.

The results of the earliest scenarios - those which simulate hot-Jupiter emplacement at $a_{\mathrm{g}} \approx 0.1 \mathrm{AU}-$ are very similar to the late scenarios of Fogg \& Nelson (2007a,b), where the evolution of the nebula is advanced and dissipative forces that stem from the presence of gas are relatively weak. Most of the inner system disk solids survive the traverse of the giant planet and most of this surviving matter is scattered into higher orbits where planet formation can resume. Water-rich habitable planets are possible within a habitable zone that is far removed from the final position of the giant planet. Where dissipative processes are enhanced by type I migration, one or two hot-Earths or hot-Neptunes are found to persist in orbits interior to the giant planet.

In "warm-Jupiter" type scenarios, where $a_{\mathrm{g}} \gg 0.1 \mathrm{AU}$, the bulk of the disk solids survive as before, but more complex systems of hot terrestrial planets interior to the giant are predicted, in larger numbers with increasing $a_{\mathrm{g}}$. If strong type I migration forces are a genuine influence on planet formation, relatively crowded systems of interior planets are predicted, although it has not been practical to check this forecast with Gyr-long integrations. Habitable planets in low eccentricity warm-Jupiter systems appear possible if the giant planet makes a limited incursion into the outer regions of the habitable zone, or traverses its entire width and keeps going until it ceases migrating at a radial distance of less than half that of the HZ's inner edge: i.e. the giant planet's final orbit lies outside the region of $\sim 0.3-1.5$ AU for a $1 M_{\odot}$ star. If giant planets strand within this region, the presence of habitable planets remains a possibility if they are located in a stable 1:1 resonance with the giant. These findings should hold for stars with different luminosities with an appropriate scaling of radial distance.

However, warm-Jupiter type exoplanets with near-circular orbits, i.e. $e \lessgtr 0.05$, are relatively uncommon and $e$ is typically observed to be considerably higher. Many of these exoplanets 


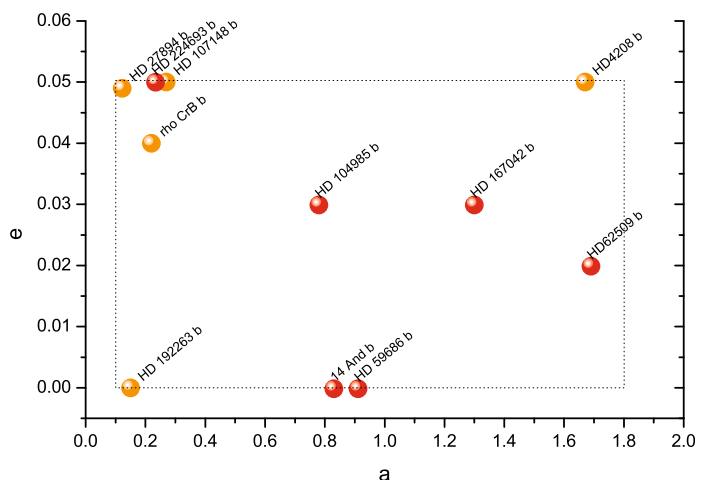

Fig. 15. Data of low eccentricity exoplanets using data from the Extrasolar Planets Encyclopedia: http: //exoplanet. eu. Plotted objects correspond to the type of warm-Jupiters within the remit of the model presented here: solitary giant planets orbiting single stars with $0.1 \mathrm{AU}<a<1.8 \mathrm{AU}, e<0.05$. Orange symbols represent exoplanets within main sequence star systems; red symbols represent exoplanets orbiting post-main sequence stars.

may have had an origin involving mutual giant planet scattering, perhaps combined with migration (e.g. Ford et al. 2001; Papaloizou \& Terquem 2001; Moorhead \& Adams 2005), as opposed to the strongly damped pure type II migration mechanism adopted here. Nevertheless, low- $e$ and solitary warm-Jupiters are known, which presumably could have originated in a similar manner to that simulated here. Figure 15 uses data from the Extrasolar Planets Encyclopedia to plot exoplanetary data between $a=0.1-1.8$ AU vs. $e=0-0.05$ which might be regarded as lying within the remit of the model. Solitary giant planets around single stars are plotted and labelled with their names. Four of these exoplanets, $\rho \mathrm{CrB}$ b, HD $27894 \mathrm{~b}$, HD $192263 \mathrm{~b}$ and HD $4208 \mathrm{~b}$ occur in systems where there is dynamical room for terrestrial planets in the habitable zone according to the stability calculations of Jones et al. (2005, 2006). Systems containing the exoplanets HD 224693 b (Jones et al. 2006), HD 104985 b and HD 59686 b (Jones et al. 2005) could also have hosted planets in their habitable zones in the past before their primaries left the main sequence. According to the results of the model presented in this paper, it appears feasible that habitable planets could have originated in these systems as well.

\section{References}

Adams, F. C., \& Laughlin, G. 2003, Icarus, 163, 290

Alexander, R. D., Clarke, C. J., \& Pringle, J. E. 2006, MNRAS, 369, 229

Armitage, P. J. 2003, ApJ, 582, L47

Armitage, P. J. 2007, ApJ, 665, 1381

Armitage, P. J., Livio, M., Lubow, S. H., \& Pringle, J. E. 2002, MNRAS, 334, 248

Asphaug, E., Agnor, C., \& Williams, Q. 2006, Nature, 439, 155

Bertout, C. 1989, ARA\&A, 27, 351

Boss, A. P. 2000, ApJ, 536, L101

Butler, P., Wright, J. T., Marcy, G. W., et al. 2006, ApJ, 646, 505

Canup, R. M., \& Pierazzo, E. 2006, LPI, 37, 2146

Chambers, J. E. 1999, MNRAS, 304, 793
Clarke, C. J., Gendrin, A., \& Sotomayor, M. 2001, MNRAS, 328, 485

Cresswell, P., \& Nelson, R. P. 2006, A\&A, 450, 833

Cresswell, P., \& Nelson, R. P. 2008, A\&A, 482, 677

Daisaka, J. K., Tanaka, H., \& Ida, S. 2006, Icarus, 185, 492

Dvorak, R., Pilat-Lohinger, E., Schwarz, R., \& Freistetter, F. 2004, A\&A, 426, L37

Erdi, B., \& Sandor, Z. 2004, CeMDA, 92, 113

Fogg, M. J. 2008, Terrestrial planet formation in exoplanetary systems, Ph.D. Thesis (University of London), 103

Fogg, M. J., \& Nelson, R. P. 2005, A\&A, 441, 791

Fogg, M. J., \& Nelson, R. P. 2006, Intnl. J. Astrobiol., 5, 199

Fogg, M. J., \& Nelson, R. P. 2007a, A\&A, 461, 1195

Fogg, M. J., \& Nelson, R. P. 2007b, A\&A, 472, 1003

Ford, E. B., Havlickova, M., \& Rasio, F. A. 2001, Icarus, 150, 303

Genda, H., \& Abe, Y. 2005, Nature, 433, 842

Goldreich, P., \& Sari, R. 2003, ApJ, 585, 1024

Goldreich, P., Lithwick, Y., \& Sari, R. 2004, ApJ, 614, 497

Haisch, K. E., Lada, E. A., \& Lada, C. J. 2001, ApJ, 553, L153

Hayashi, C. 1981, Prog. Theor. Phys. Suppl., 70, 35

Hollenbach, D., Johnstone, D., Lizano, S., \& Shu, F. 1994, ApJ, 428, 654

Jones, B. W., Underwood, D. R., \& Sleep, P. N. 2005, ApJ, 622, 1091

Jones, B. W., Sleep, P. N., \& Underwood, D. R. 2006, Intnl. J. Astrobiol., 5, 25

Kasting, J. F., Whitmire, D. P., \& Reynolds, R. T. 1993, Icarus, 101, 108

Kokubo, E., \& Ida, S. 2000, Icarus 143, 15

Kuchner, M. J., \& Lecar, M. 2002, ApJ, 574, L87

Léger, A., Selsis, F., Sotin, C., et al. 2004, Icarus, 169, 499

Lin, D. N. C., \& Ida, S. 1997, ApJ, 477, 781

Lin, D. N. C., \& Papaloizou, J. C. B. 1986, ApJ, 309, 846

Lin, D. N. C., Bodenheimer, P., \& Richardson, D. C. 1996, Nature, 380, 606

Lineweaver, C. H. 2001, Icarus, 151, 307

Lineweaver, C. H., Fenner, Y., \& Gibson, B. K. 2004, Science, 303, 59

Lissauer, J. J. 1987, Icarus, 69, 249

Mandell, A. M., Raymond, S. N., \& Sigurdsson, S. 2007, ApJ, 660, 823

Marzari, F., \& Weidenschilling, S. J. 2002, Icarus, 156, 570

Masset, F. S., Morbidelli, A., Crida, A., \& Ferreira, J. 2006, ApJ, 642, 478

McArthur, B. E., Endl, M., Cochran, G., et al. 2004, ApJ, 614, L81

McNeil, D., Duncan, M., \& Levison, H. F. 2005, AJ, 130, 2884

Moorhead, A. V., \& Adams, F. C. 2005, Icarus, 178, 517

Moorhead, A. V., \& Adams, F. C. 2008, Icarus, 193, 475

Nelson, R. P., Papaloizou, J. C. B., Masset, F. S., \& Kley, W. 2000, MNRAS, 318, 18

Ogilvie, G. I., \& Lubow, S. H. 2003, ApJ, 587, 398

Papaloizou, J. C. B. 2007, A\&A, 463, 775

Papaloizou, J. C. B., \& Larwood, J. D. 2000, MNRAS, 315, 823

Papaloizou, J. C. B., \& Nelson, R. P. 2005, A\&A, 433, 247

Papaloizou, J. C. B., \& Terquem, C. 2001, MNRAS, 325, 221

Papaloizou, J. C. B., Nelson, R. P., \& Masset, F. 2001, A\&A, 366, 263

Pollack, J. B., Hubickyj, O., Bodenheimer, P., et al. 1996, Icarus, 124, 62

Raymond, S. N., Quinn, T., \& Lunine, J. I. 2004, Icarus, 168, 1

Raymond, S. N., Mandell, A. M., \& Sigurdsson, S. 2006, Science, 313, 1413

Raymond, S. N., Quinn, T., \& Lunine, J. I. 2007, AsBio, 7, 66

Raymond, S. N., Barnes, R., Armitage, P. J., \& Gorelick, N. 2008, ApJ, 687, L107

Reynolds, R. T., Squyres, S. W., Colburn, D. S., \& McKay, C. P. 1983, Icarus, 56,246

Reynolds, R. T., McKay, C. P., \& Kasting, J. F. 1987, AdSpR, 7, 125

Selsis, F., Kasting, J. F., Levrard, B., et al. 2007, A\&A, 476, 1373

Simon, M., \& Prato, L. 1995, ApJ, 450, 824

Takeuchi, T., Miyama, S. N., \& Lin, D. N. C. 1996, ApJ, 460, 832

Tanaka, H., \& Ward, W. R. 2004, ApJ, 602, 388

Tanaka, H., Takeuchi, T., \& Ward, W. R. 2002, ApJ, 565, 1257

Thommes, E. W., Duncan, M. J., \& Levison, H. F. 2003, Icarus, 161, 431

Trilling, D. E., Benz, W., Guillot, T., et al. 1998, ApJ, 500, 428

von Bloh, W., Bounama, C., Cuntz, M., \& Franck, S. 2007, A\&A, 476, 1365

Ward, P. D., \& Brownlee, D. 2000, Rare Earth: Why complex life is uncommon in the universe (New York: Copernicus Books)

Ward, W. R. 1997, Icarus, 126, 261

Wolk, S. J., \& Walter, F. M. 1996, AJ, 111, 2066 